Case Report

\title{
Incidental Intracranial Aneurysm in a Dog Detected by 16-Multidetector Row Computed Tomography Angiography
}

\author{
Giovanna Bertolini \\ San Marco Veterinary Clinic, Diagnostic Imaging Division, Via Sorio 114/c, 35141 Padova, Italy \\ Correspondence should be addressed to Giovanna Bertolini; bertolini@sanmarcovet.it
}

Received 10 December 2012; Accepted 8 January 2013

Academic Editors: C. Gutierrez, S. C. Rahal, and S. Stuen

Copyright (C) 2013 Giovanna Bertolini. This is an open access article distributed under the Creative Commons Attribution License, which permits unrestricted use, distribution, and reproduction in any medium, provided the original work is properly cited.

\begin{abstract}
This paper describes a small intracranial aneurysm incidentally found in a 24-month-old Nova Scotia Duck Tolling Retriever evaluated for a recent history of lethargy, fever, and cervical pain. The clinicopathological analysis revealed leukocytosis, and increased haptoglobin and C-reactive protein consistent with severe flogistic process. Nonenhanced computed tomography of the brain and cervical spine showed a diffuse encephalopathy and moderate cervical syringohydromyelia. Computed tomography angiography series of the brain showed a small saccular dilation at the joining point of the two rostral cerebral arteries consistent with a small aneurysm. Cerebrospinal fluid examination led to the final diagnosis of aseptic meningitis. The dog was discharge with a long-term corticosteroid therapy for the meningitis. At two-month follow-up evaluation, the cerebrospinal fluid examination was normal and the computed tomography of the brain showed no abnormalities except for the stable aneurysm. To our knowledge, this is the first description of a spontaneous cerebral aneurysm in dogs and serves to broaden the spectrum of cerebrovascular diseases in this species.
\end{abstract}

\section{Introduction}

A brain aneurysm is an abnormal, outward pouching of the artery wall caused by a weakness in the wall of an artery that supplies the brain. In humans, the prevalence of this condition is about $5 \%$. Approximately, $85 \%$ of aneurysms develop in the anterior portion of the circulation of the brain and are asymptomatic until they rupture [1-3]. Cerebral aneurysms are classified based on a number of features including etiology, size, shape, the association with the specific intracranial branch, or according to their angioarchitecture features $[2,4-$ $6]$.

To date, very little is known concerning the type and incidence of variants and anomalies of the cerebrovascular system in dogs and cats [7-9]. Historically, few cases of cerebral hemorrhage in dogs were thought to be correlated to aneurysm, but their existence could not be proved [10]. To the authors knowledge, this is the first description of a spontaneous cerebral aneurysm in dogs.

\section{Case Presentation}

A 24-month-old $22 \mathrm{~kg}$ intact male Nova Scotia Duck Tolling Retriever was evaluated for a recent history of lethargy, fever, and cervical pain. Medical history included another episode of cervical pain that has occurred one year before and rapidly improved with corticosteroid therapy for few days. At the time of presentation, the dog was receiving amoxicillin/ clavulanic acid for 2 days (10 mg/kg PO q12).

At physical examination, the dog showed ventroflexion of the neck and was tachypneic ( $80 \mathrm{breath} / \mathrm{min})$, with $39.3^{\circ} \mathrm{C}$ rectal temperature and normal heart rate (96 beats/min), increased blood pressure (120-180 $\mathrm{mmHg}$, mean $145 \mathrm{mmHg}$ ), and normal mucous membrane appearance and capillary refill time (approximately $1 \mathrm{sec}$ ). Heart and thoracic auscultation were normal. Abdominal palpation revealed splenomegaly.

At neurological examination, the dog had cervical rigidity and cervical pain. Cranial nerve function, postural reaction testing, and segmental spinal reflexes were normal. The CBC showed leukocytosis accompanied by a "left shift" in the ratio of immature to mature neutrophils. Serum biochemistry profile showed increased haptoglobin and C-reactive protein. These clinicopathological results indicated a severe flogistic process.

Thoracic and cervical radiographs were normal. Abdominal ultrasound confirmed a diffuse subjective enlargement 


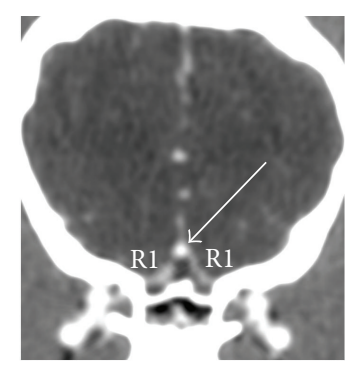

(a)

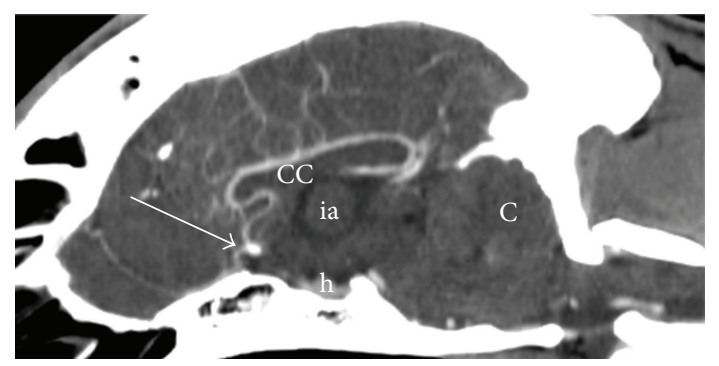

(b)

FIGURE 1: Multiplanar reformatted images (MPRs) of the neurocranium. (a) Transverse (slight oblique) view showing the small aneurysm (arrow) at the joining point of the two R1 segments of the left and right rostral cerebral arteries. (b) Mid-sagittal view of the same dog. The arrow indicates the aneurysm. CC: corpus callosum; ia: interthalamic adhesion; C: cerebellum; h: hypophysis.

of the spleen, but no other anomalies were noted. The dog was then placed under general anesthesia and underwent 16multidetector-CT (GE Lightspeed 16, GE Medical Systems, Milwaukee, WI) examination of the neurocranium, cervical spine, and abdomen. For the neurocranium, unenhanced scans were obtained followed by an angiographic series. The acquisition, dose, and reconstruction parameters were axial modality, 2 s/rotation, caudal-to-rostral direction, $16 \times$ $0.625 \mathrm{~mm}$ detector configuration, $120 \mathrm{kVp}, 210 \mathrm{mAs}$, standard algorithm. For brain CTA, $640 \mathrm{mg} \mathrm{I} / \mathrm{kg}$ of iodixanol (Visipaque320, Amersham Health, Princeton, HJ) was injected at $37^{\circ} \mathrm{C}$ at a rate of $4 \mathrm{~mL} / \mathrm{s}$ using a power injector system, (Stellant, Medrad, Indianola, IA) through a 20 gauge catheter placed in the left cephalic vein. An injection-to-scan delay of $10 \mathrm{sec}$. was used. Original data sets of $512^{2}$ matrix size were transferred to a freestanding workstation (Advantage Workstation 4.1, GE Medical Systems, Milwaukee, WI) and postprocessed using multiplanar reformation (MPR), maximum intensity projection (MIP), and volume rendering (VR) techniques. Unenhanced $\mathrm{CT}$ of the neurocranium revealed a moderate diffuse hypoattenuation of the white matter. In the cervical spine, a slight dilation of the central canal was noted. These CT features were consistent with a diffuse encephalopathy and moderate cervical syringohydromyelia. The CTA series of the brain showed the two rostral cerebral arteries anastomosed with each other rostro-dorsally to the optic chiasm forming a single median artery. At the joining point there was a small saccular dilation, enhancing at the same degree of the arteries, consistent with a small aneurysm (Figures 1, 2, and 3). Measurements of the aneurysm were

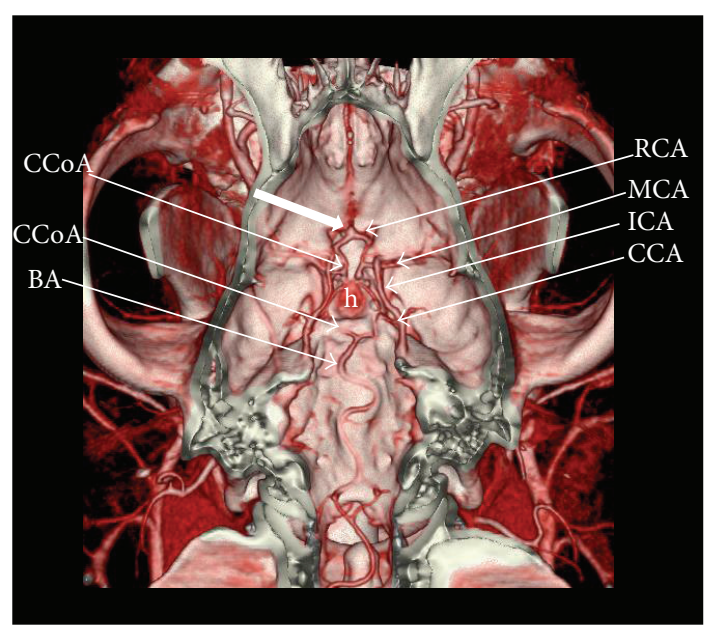

FIGURE 2: Volume rendering (VR) of the skull, dorsal view. The vault of the skull has been removed to visualize the circulus arteriosus cerebralis (Circle of Willis). RCA: rostral cererbal artery; MCA: middle cerebral artery; ICA: internal carotid artery; CCA: caudal cerebral artery; CCoA: caudal communicating artery; BA: basilar artery; h: hypophysis. The arrow indicates the small aneurysm at the joining point of the two rostral cerebral arteries (RCAs).

performed from magnified MPR images with an electronic caliper at the workstation. The aneurysm was $1.3 \mathrm{~mm}$ at the neck point, $2.0 \mathrm{~mm}$ in width, and $1.8 \mathrm{~mm}$ in height (Figure 4).

Contrast-enhanced abdominal CT revealed a saccular dilation of the prehepatic segment of the portal vein and a small dilation of the umbilical part of the left portal branch within the liver. These features were consistent with extrahepatic and intrahepatic portal vein aneurysms.

Cisternal cerebrospinal fluid (CSF) was collected at the end of the CT scans. CSF had 324 white blood cells/ $\mu \mathrm{L}$ (reference range, $\leq 5$ cells $/ \mu \mathrm{L}$ ), 142 red blood cells $/ \mu \mathrm{L}$, and $21 \mathrm{mg} / \mathrm{dL}$ protein (reference range, $\leq 25$ ). Glucose concentration was $76 \mathrm{mg} / \mathrm{dL}$ (reference range, 53-104 mg/dL). The differential cell count indicated a neutrophilic and macrophagic pleocytosis (80\% neutrophils and 20\% macrophages).

Based on these findings, the final diagnosis was of aseptic meningitis. The dog was discharged with a long-term corticosteroid therapy. The owner reported that the dog had a rapid improvement, and at two-month follow-up evaluation, the cerebrospinal fluid examination was normal. CT of the neurocranium showed the rostral cerebral small aneurysm stable. The enhanced abdominal scan showed a large filling defect in the aneurysmal portal vein, consistent with thrombosed portal vein aneurysm. The small intrahepatic aneurysm was stable. Followup by CT scan and/or US of the abdomen was suggested.

\section{Discussion}

The overall schema of the blood supply to the cranial region and the brain, the histological structure of the walls of the arteries, and the basic pattern of the embryological development are essentially the same in dog as they are in man (Figure 5) [7, 11]. Many variations of the Circle of Willis have 


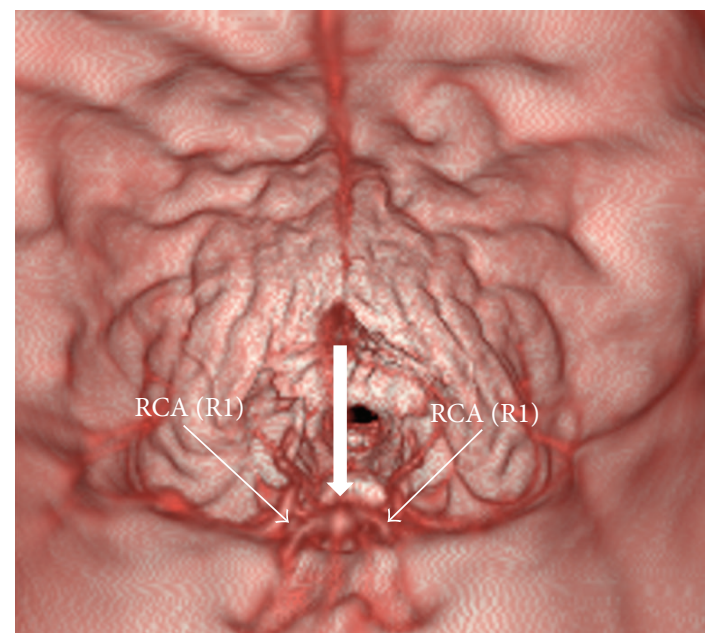

(a)

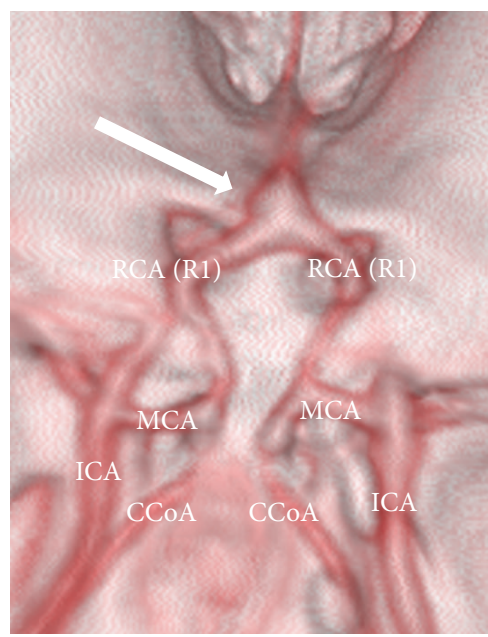

(b)

FIGURE 3: Virtual navigation through the cranial cavity. (a) Forward view, showing the small saccular aneurysm at the joining point of the two pre-joining segments (R1) of the left and right rostral cerebral arteries. (b) Dorsal backward view of the Circle of Willis showing the small aneurysm (arrow). RCA: rostral cerebral artery; R1: prejoining segment; MCA: middle cerebral artery; ICA: internal carotid artery; CCoA: caudal communicating artery.

been described in humans, and they play a significant role in the cerebral aneurysms formation, in terms of hemodynamic stress caused by variations $[12,13]$. In contrast, variants of the Circle of Willis have been rarely described in dog so far $[7,14,15]$. In most dogs, the two rostral arteries form a common median trunk, as described in the present case.

In humans, various shear stresses (flow, turbulences, jet effects, and others) are known to produce aneurysms. These represent "luminal" aneurysmal vasculopathies in which it is postulated that these stresses induce pathogenetic changes in normal vessel walls. In contrast, structural vessel-wall diseases (inflammatory, infectious, collagen diseases, and others) are "abluminal" aneurysmal vasculopathies. In these,

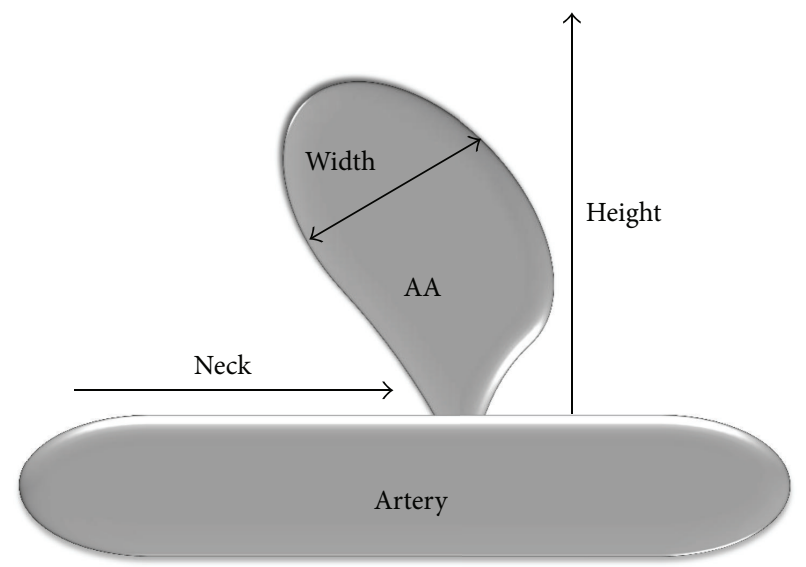

Figure 4: Drawing showing the measurement technique of the saccular aneurysm (AA).

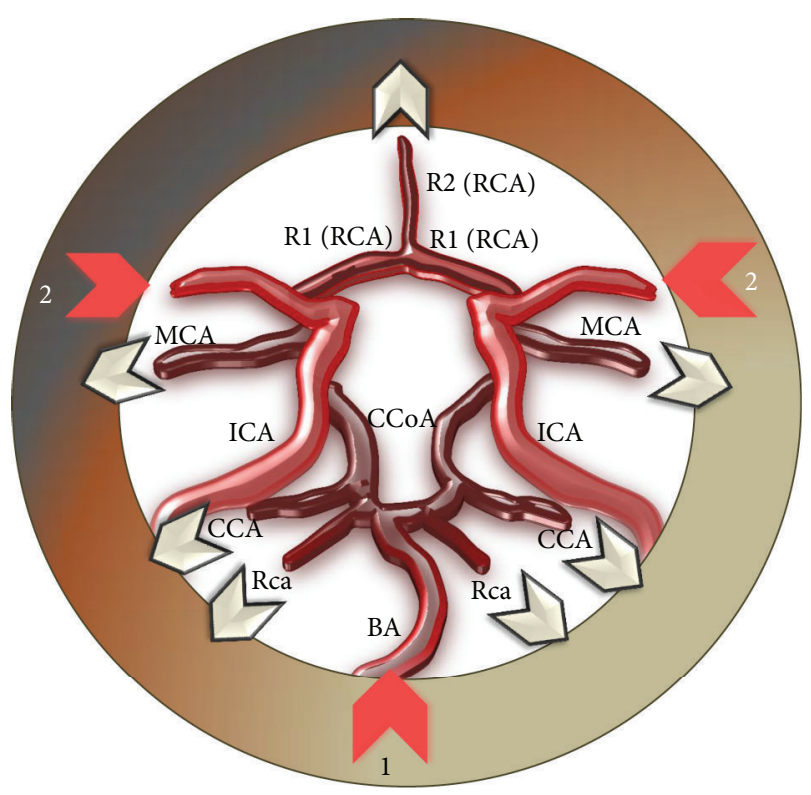

Figure 5: The drawing shows the blood flow to the Circle of Willis from the three vessels: 1, basilar artery (BA), and 2, internal carotid arteries (ICAs). From internal carotid arteries, blood flows from circle of Willis to the brain through the rostral cerebral arteries (RCA). The anterior communicating artery that connects the two anterior cerebral arteries in humans is rarely described in dogs. However, either the canine rostral cerebral artery can be subdivided into a pre-joining point (R1) and, generally, in a single median R2 segment (post-joining). Internal carotid artery gives off the median cerebral arteries (MCAs) and the caudal communicating arteries (CCoAs), that connect the circle with the basilar artery. In most dogs, the caudal cerebral artery (CCA) and the rostral cerebellar artery (RcAs) are from the caudal communicating artery.

there is a primary abnormality of the vessel wall which is potentially aggravated by shear stresses [1, 3-6].

From the clinical point of view, most human patients have no symptoms or complaints until the aneurysm ruptures. Neurological signs in our patient were attributed to the meningitis. The meningitis of our Nova Scotia Duck 
Tolling Retriever was diagnosed as aseptic meningitis, a noninfectious inflammatory disorder frequently diagnosed in this breed of dog [16]. Aetiological hypotheses of the cerebral aneurysm in our dog include congenital weakness or degenerative changes in vessel walls due to a connective tissue disorder or a local inflammatory process. This dog had simultaneous extrahepatic and intrahepatic portal vein aneurysms. Portal vein aneurysm is a rare condition we recently described in a series of dogs including the dog of this paper [17]. Aneurysm of the portal vein can be either congenital or acquired and thrombosis of the aneurysmal portal vein with its consequences is a possible complication. The development of more than one aneurysm at different site is an uncommon event in human patients $[18,19]$ and it has been reported once in a dog [20].

This incidentally discovered cerebral aneurysm broads the spectrum of cerebrovascular diseases in dogs. Intracranial vessels can now be routinely assessed in living animals using $\mathrm{CT}$ and magnetic resonance (MR) imaging [21-24]. With the widespread use of these advanced imaging technologies, the potential to identify variants of the Circle of Willis or anomalies of the cerebrovascular system in dogs can substantially increase. Moreover, the non- or minimally invasive nature of these techniques, combined with the ability to obtain information about the brain parenchyma within the same examination, represents a clear advantage of CT-angiography (CTA) and MR angiography (MRA) over all other imaging techniques [21-23].

\section{References}

[1] T. Krings, D. M. Mandell, T. R. Kiehl et al., "Intracranial aneurysms: from vessel wall pathology to therapeutic approach," Nature Reviews Neurology, vol. 7, no. 10, pp. 547-559, 2011.

[2] M. B. Pritz, "Cerebral aneurysm classification based on angioarchitecture," Journal of Stroke and Cerebrovascular Diseases, vol. 20, no. 2, pp. 162-167, 2011.

[3] I. Loumiotis, A. Wagenbach, R. D. Brown Jr., and G. Lanzino, "Small $(<10-\mathrm{mm})$ incidentally found intracranial aneurysms, part 1: reasons for detection, demographics, location, and risk factors in 212 consecutive patients," Neurosurg Focus, vol. 31, no. 6, p. E3, 2011.

[4] F. Bonneville, N. Sourour, and A. Biondi, "Intracranial aneurysms: an overview," Neuroimaging Clinics of North America, vol. 16, no. 3, pp. 371-382, 2006.

[5] M. A. Castro, C. M. Putman, M. J. Sheridan, and J. R. Cebral, "Hemodynamic patterns of anterior communicating artery aneurysms: a possible association with rupture," American Journal of Neuroradiology, vol. 30, no. 2, pp. 297-302, 2009.

[6] T. J. Grobelny, "Brain aneurysms: epidemiology, treatment options, and milestones of endovascular treatment evolution," Disease-a-Month, vol. 57, no. 10, pp. 647-655, 2011.

[7] K. Kapoor, V. K. Kak, and B. Singh, "Morphology and comparative anatomy of circulus arteriosus cerebri in mammals." Anatomia, Histologia, Embryologia, vol. 32, no. 6, pp. 347-355, 2003.

[8] W. R. Hause, M. L. Helphrey, R. W. Green, and P. C. Stromberg, "Cerebral arteriovenous malformation in a dog," Journal of the American Animal Hospital Association, vol. 18, pp. 601-607, 1982.
[9] W. B. Thomas, R. O. Scheuler, and J. N. Kornegay, "Surgical excision of a cerebral arteriovenous malformation in a dog," Progress in Veterinary Neurology, vol. 6, pp. 20-23, 1995.

[10] J. T. McGrath, Neurologic Examination of the Dog with ClinicoPathologic Observations, Lea \& Febiger, Philadelphia, Pa, USA, 2nd edition, 1960.

[11] O. Shaller, Illustrated Veterinary Anatomic Nomenclature, Georg Thieme, 2nd edition, 2007.

[12] A. F. van Raamt, W. P. Mali, P. J. van Laar, and Y. van der Graaf, "The fetal variant of the circle of Willis and its influence on the cerebral collateral circulation.," Cerebrovascular Diseases, vol. 22, no. 4, pp. 217-224, 2006.

[13] K. Kapoor, B. Singh, and L. I. J. Dewan, "Variations in the configuration of the circle of Willis," Anatomical Science International, vol. 83, no. 2, pp. 96-106, 2008.

[14] W. E. Stehbens, "Cerebral aneurysms of animals other than man," The Journal of Pathology and Bacteriology, vol. 86, pp. $160-168,1963$.

[15] R. Frankhauser, H. Luginbuhl, and J. T. McGrath, "Cerebrovascular disease in various animal species," Annals of the New York Academy of Sciences, vol. 127, no. 1, pp. 817-860, 1965.

[16] K. P. Anfinsen, M. Berendt, F. J. H. Liste et al., "A retrospective epidemiological study of clinical signs and familial predisposition associated with aseptic meningitis in the Norwegian population of Nova Scotia duck tolling retrievers born 19942003," Canadian Journal of Veterinary Research, vol. 72, no. 4, pp. 350-355, 2008.

[17] G. Bertolini and M. Caldin, "Computed tomography findings in portal vein aneurysm of dogs," The Veterinary Journal, vol. 193, no. 2, pp. 475-480, 2012.

[18] W. J. Henry, "Multiple aneurysm formation in a young man: a case report," Annals of surgery, vol. 158, pp. 1043-1046, 1963.

[19] Y. Ito, K. Tarao, S. Tamai et al., "Portal vein aneurysm in the liver associated with multiple vascular malformations," Journal of Gastroenterology, vol. 29, no. 6, pp. 776-781, 1994.

[20] K. R. Salmeri, J. R. Bellah, N. Ackerman, and B. Homer, "Unilateral congenital aneurysm of the jugular, linguofacial, and maxillary veins in a dog," Journal of the American Veterinary Medical Association, vol. 198, no. 4, pp. 651-654, 1991.

[21] M. Sager, J. Assheuer, H. Trümmler, and K. Moormann, “Contrast-enhanced magnetic resonance angiography (CE-MRA) of intra- and extra-cranial vessels in dogs," Veterinary Journal, vol. 179, no. 1, pp. 92-100, 2009.

[22] P. Martin-Vaquero, R. C. da Costa, R. L. Echandi, C. L. Tosti, M. V. Knopp, and S. Sammet, "Time-of-flight magnetic resonance angiography of the canine brain at 3.0 Tesla and 7.0 Tesla," American Journal of Veterinary Research, vol. 72, no. 3, pp. 350356, 2011.

[23] A. S. Tidwell and I. D. Robertson, "Magnetic resonance imaging of normal and abnormal brain perfusion," Veterinary Radiology and Ultrasound, vol. 52, no. 1, supplement 1, pp. S62-S71, 2011.

[24] O. D. Jacqmot, F. R. Snaps, N. M. Maquet, M. P. Heinen, and A. E. Gabriel, "Arterial head vascularization cartographies of normal metencephalic dogs using magnetic resonance angiography," The Anatomical Record, vol. 294, no. 11, pp. 1834-1841, 2011. 

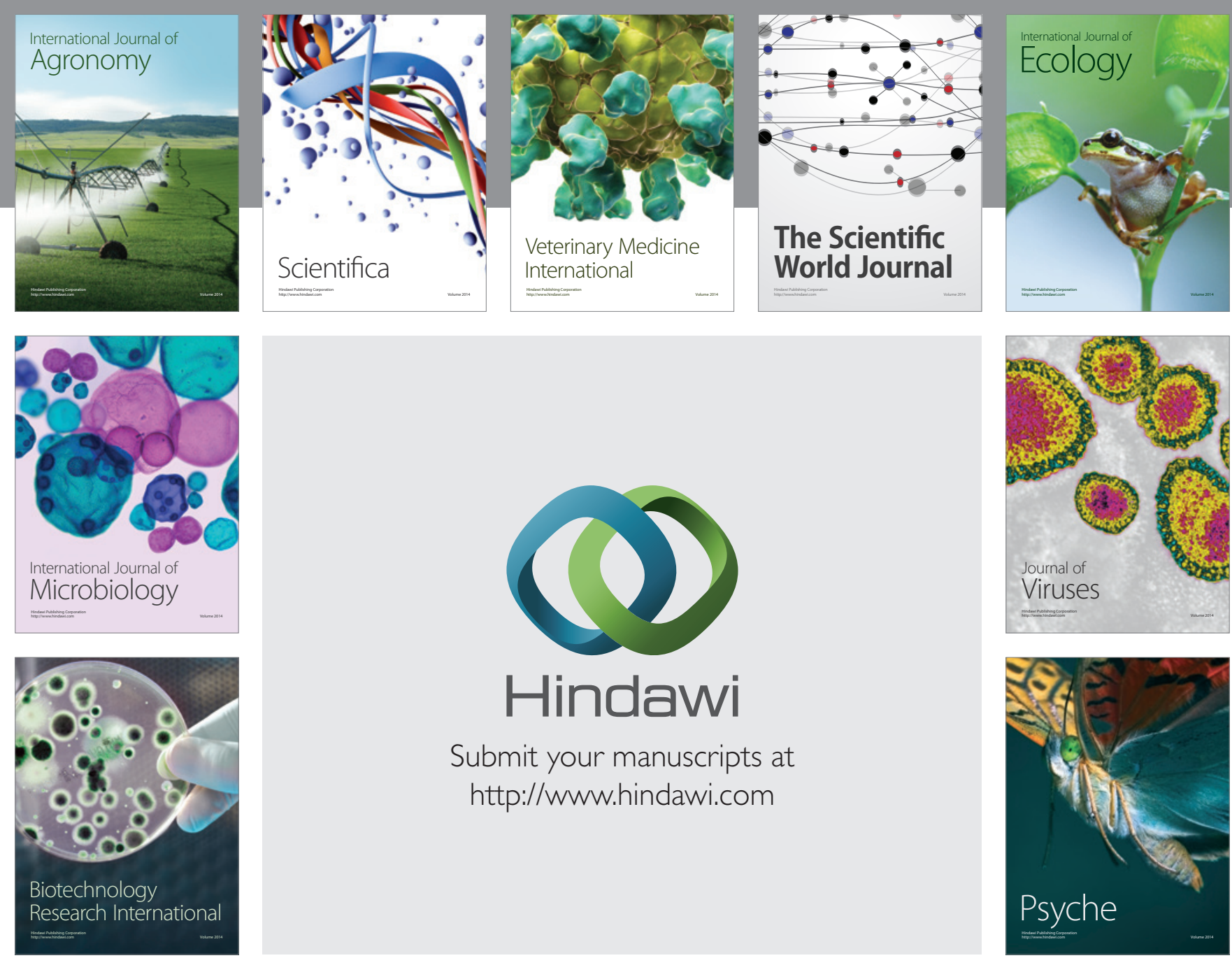

Submit your manuscripts at http://www.hindawi.com
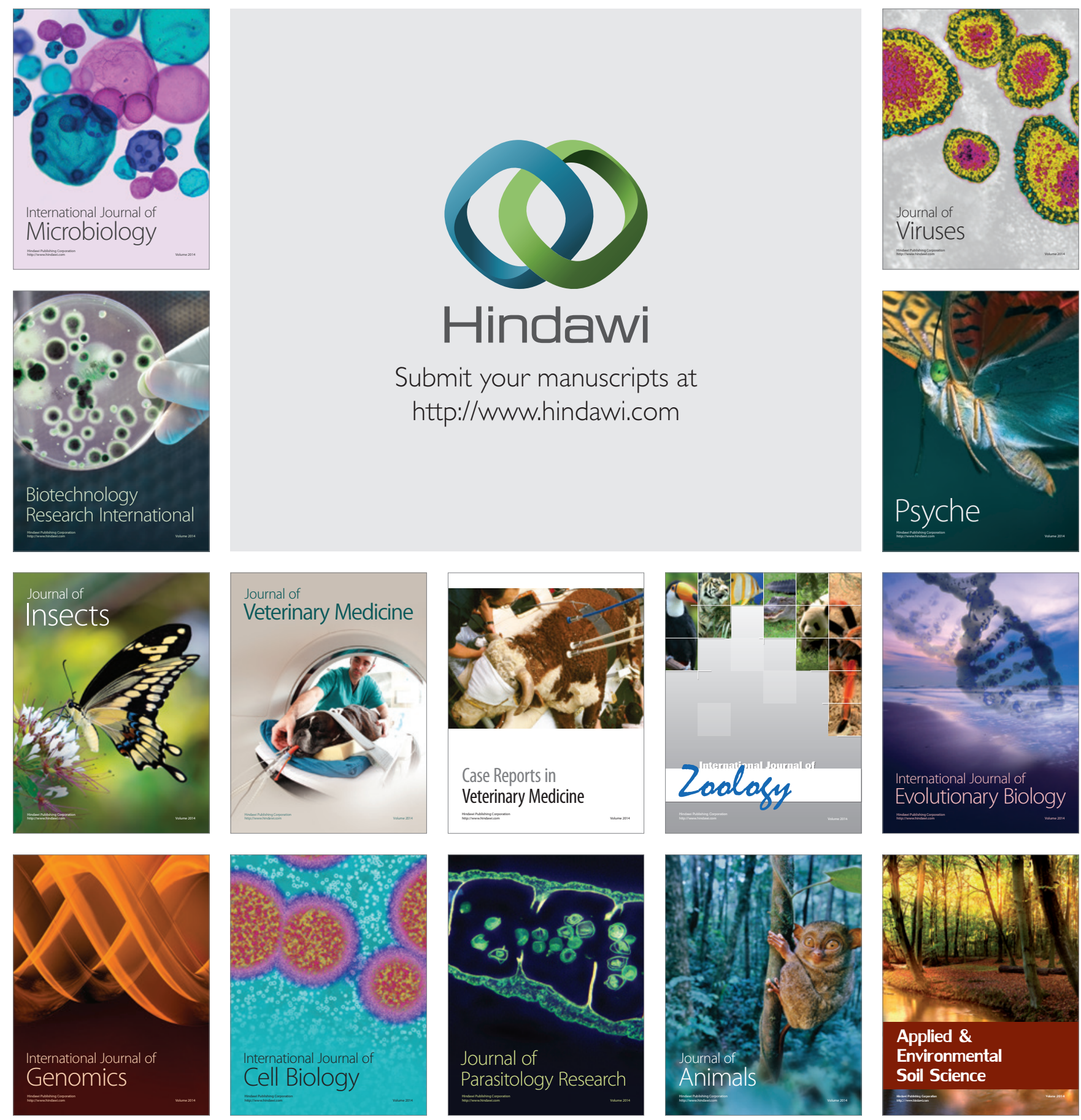